\title{
Konflikte im Kirchenfernsehen - Zum Beispiel „Blickfeld”
}

\author{
von Konrad Simons
}

„Verbreite die Lehren deiner Religion über die ganze Welt, töte und schlachte diejenigen, die gegen dich sind, nimm dir Land, um Kirchen darauf zu bauen." Mit diesen Worten kennzeichnete an einem Samstagnachmittag ${ }^{1}$ das „Blickfeld - Kirche und Gesellschaft" im (ersten) ARD-Fernsehen die Arbeit christlicher Missionare. Ein indianischer Protestsänger trug seine Ansichten auf einer Kundgebung für bedrohte Völker vor. In deutscher Übersetzung eingeblendet hieß es weiter: „Nimm ein Kind, solange es noch erziehbar ist, verdirb sein Denken und seine Empfindungen. Geh und erzähl dem Eingeborenen, daß er Christ werden muß . . . Stopf ihm die Heilige Schrift in den Rachen, würg ihm deine Worte rein, bis es ihm den Hals zuschnürt!"

Der Moderator der Sendung, zugleich verantwortlicher „Blickfeld”-Redakteur des Westdeutschen Rundfunks ${ }^{2}$, stellte fest, daß in den Kirchen „ein Umdenken im Gange” sei; in Brasilien schütze die katholische Kirche die Indianer. Er distanzierte sich nicht von der Behauptung des Protestsängers, daß Missionare morden, stehlen, ausbeuten, terrorisieren.

\section{Konfliktstoffe}

Ist die zitierte Sendung typisch für das Kirchenfernsehen der Arbeitsgemeinschaft der Rundfunkanstalten Deutschlands (ARD)?

Um dieser Frage nachzugehen, wurden von Januar 1978 an alle der grundsätzlich einmal im Monat ausgestrahlten „Blickfeld”-Sendungen analysiert. In insgesamt 14 Sendungen gab es 43 Beiträge. Von ihnen waren 40 als Konfliktstoffe einzustufen. Die meisten Sendungen befaßten sich mit der evangelischen Kirche. 18 der Einzelstreifen waren protestantisch, 8 katholisch, 8 bikonfessionell, 1 jüdisch, der Rest sozialkritischungebunden.

\section{Katholiken}

„Ein Priester geht stempeln” war das Stichwort eines Beitrags, der sich mit der Entlassung des Spanienseelsorgers Don José in Bochum befaßte und Zusammenhänge zu sehen glaubte zwischen des Pfarrers Kritik an Adveniat und Misereor und der angeblichen Hergabe von Geldern an militärische Diktaturen in Südamerika ${ }^{3}$. Zu Wort kam nur die „Basis”. Dem Streifen voraus lief ein Film „Die Kirche im Dorf - priesterlos aber glücklich" 4 , der laut Ansage auch untersuchte, ob der Priestermangel den Zölibat untergrabe. Die Zuordnung zum stempelnden Priester in Bochum war gezielt.

„Katholische Kirche und Homosexualität” war das Thema einer Sendung, die den Moderator zu der Feststellung veranlaßte, daß es „für die Römische Glaubenskongregation keine Homosexuellen in der Kirche gibt, weil es sie nicht geben darf" 5 . Am Beispiel Holland wurde Seelsorge für Homophile aufgezeigt. Moraltheologen und „Amtskirche” kamen nicht zu Wort. Auch hier ein Zusammenhang mit dem zweiten Streifen „Rassismus in Deutschland"6. Verpönung hier wie dort?

Dr. iur. Konrad Simons war lange Jahre Redakteur der „Rheinischen Post”, Düsseldorf, Chefredakteur der „Aachener Volkszeitung”, Vorsitzender der Gesellchaft Katholischer Publizisten Deutschlands und ist stellv. Mitglied des WDR-Rundfunkrats. 
Demselben Verfasser wurde Gelegenheit ${ }^{7}$ gegeben, die Sonder-Rollen der Evangelischen Kirche in Deutschland (EKD) bei der Bekämpfung des Rassismus in Südafrika innerhalb des Ökumenischen Rates in Genf zu begründen. Dürfen kirchliche Mittel für die Versorgung der Befreiungsbewegungen mit Waffen verwandt werden? Der Konflikt der EKD schimmerte zugunsten Genfs von den deutschen Mattscheiben.

Offensichtlich auf Ausgewogenheit bedacht, brachte ein „Blickfeld”8 Billy Grahams Polenreise gemeinsam mit Bildern von der Ortschaft der Geburt des neuen PolenPapstes - beides gute Reportagen. Köntrovers dazu ein Beitrag über den Konflikt der Katholischen Jugend mit der deutschen Bischofskonferenz, einseitig zugunsten der „Basis"9.

Unter dem Motto „Kirchen und Christen im Sozialismus Angolas” 10 wurde vom WDRRedakteur behauptet, daß die katholische Kirche seit der Beendigung der portugiesischen Herrschaft „ihre Privilegien” eingebüßt habe. Mehr Tendenz verhieß das Eingangsbild mit folgendem Text: „Eine Kapelle an der Küste Angolas, in der Nähe der Hauptstadt Luanda. Hier gab die Kirche den Sklavenschiffen nach Amerika bis ins 19. Jahrhundert hinein ihren Segen.” Und weiter: „Am Ende segnete sie die Panzer, mit denen die portugiesische Diktatur als letztes europäisches Land versuchte, ihre Kolonialherrschaft aufrechtzuerhalten."

Dem farbigen Methodistenbischof Carvalho standen Kamera und Mikro zur Verfügung, um zu erklären, daß die Kirche unter der neuen Verfassung ihre Freiheit genieße. Auf die Frage, warum die katholischen Oberhirten in ihrem Hirtenbrief die Lage der Kirche bedauern, antwortete der laut „Welt" ${ }^{11}$ auf der Moskauer Friedenskonferenz sozialistisch hervorgetretene Methodist: „Die Gründe ... sehe ich in der traditionellen Weise, in der die katholische Kirche den wissenschaftlichen Sozialismus betrachtet!" Carvalho versäumte auch nicht $z u$ bemängeln, daß die Bischöfe die katholischen Lehrer aus den Schulen abgezogen hätten, erwähnte jedoch nicht, daß von jedem Lehrer in Angola verlangt wird, den Atheismus zu lehren ${ }^{11}$.

Der Angolabeitrag hatte zu Protesten in Bevölkerung, in Kirche und in den WDRAufsichtsgremien geführt ${ }^{12}$. Missio, das internationale katholische Missionswerk in Aachen, hatte „am Stil und an der Tendenz der Sendung Anstoß genommen”. In einer Erklärung heißt es weiter: „Skandalös schien uns vor allem, daß die Fragesteller die Sicherheit ihrer Interviewpartner und die Arbeit einer ganzen Kirche leichtfertig aufs Spiel gesetzt haben." Die Interessen „unserer Partner, der katholischen Kirche in Angola", seien durch den Beitrag sträflich verletzt worden. Dem WDR-Redakteur wurde angeraten, künftig sorgfältiger zu recherchieren und daß er „,bei Themen, die die katholische Kirche in der Dritten Welt betreffen, seine Sympathien den eigentlich Betroffenen schenkt und auch das Urteil von Fachleuten einholt, die sich diese Themen zur Lebensaufgabe gemacht haben"13.

\section{Protestanten}

Auch die evangelischen Beiträge sind konfliktgeladen. Als Beispiel gewaltfreier Aktion kirchlicher Mitarbeiter in Hamburg wird für die Ablehnung der Atomenergie geworben ${ }^{14}$, kommen Arbeitsgemeinschaften der Kriegsdienstverweigerer zu Wort ${ }^{15}$ und weniger die Pfadfinder ${ }^{16}$, denen Zusammenarbeit mit Heimattreuen vorgeworfen wird. Ein Bericht über die evangelische Thomas-Gemeinde in Düsseldorf verlangte Solidarität für die Unterdrückten in Nicaragua ${ }^{17}$. Eine protestantische Frauen-Aktion, die zum 
Boykott südafrikanischer Waren aufforderte ${ }^{18}$, reklamierte Sympathien. Obwohl der WDR-Redakteur auch Gegenstimmen aus evangelischen Kreisen zu dieser Aktion zu bringen versprach, wurde bis fünf Monate nach der Zusage weder bildlich noch textlich etwas davon ausgestrahlt.

Der tödliche Unfall eines Bergmanns ließ die Frage stellen, was ein Pfarrer mit der Sicherheit am Arbeitsplatz zu tun habe ${ }^{19}$. „Dieser Tod hat Gott nicht gefallen”, sagte der Pastor am Grab. Die Werksleitung ging gerichtlich gegen ihn vor. Die Klage wurde abgewiesen unter Hinweis auf den besonderen Schutz der Religionsausübung. Der Beitrag fragte nach Konsequenzen, da der Arbeiter praktisch auf sich allein gestellt sei. „Die Arbeitgeber sperren aus!” Die evangelische Landeskirche wurde zu dieser sozialkritischen Frage nicht angegangen.

Im Rahmen des Antirassismusprogramms des Weltkirchenrates stellte „Blickfeld” einen Aktionsplan für die australischen Ureinwohner vor ${ }^{20}$. Die Australierin Cath Walker bezichtigte die englischen Eroberer, ihren eingeborenen Vorfahren nur „Bibel, Krankheiten, Alkohol und das Gewehr" gebracht und sie wie die Pest bekämpft zu haben. Angeklagt wurden die Mordtaten des weißen Mannes und das alte christliche Missionsverständnis. Geschickt war die Bildauswahl der Verfolgungszeit. Eine Muttergottesdarstellung verschwamm in ein verhungertes Mutter-Kind-Foto aus einer armseligen Eingeborenensiedlung. Man vermißte eine Äußerung der vom Moderator vorher erwähnten Mitgliedskirchen des Weltkirchenrats.

\section{Kirche im Magazin}

„Blickfeld, Kirche und Gesellschaft” ist eine gemeinsame Sendereihe aller ARD-Anstalten und wird in der Regel zehnmal im Jahr vom WDR dem ersten Programm angedient. Diese als Kirchenfunk bezeichnete Magazin-Serie wurde vom Südwestfunk zunächst viermal im Jahr besorgt und dann im Wechsel von den einzelnen Anstalten vorbereitet. Die Reihe soll die Ereignisse im kirchlichen Bereich, ohne Aktualitätszwang, wahrnehmen und die „Tagesthemen” ergänzen. Obwohl der Redaktion mehr Zeit zur Verfügung steht als den „Aktuellen”, kommt es immer wieder zu Pannen. So wurden nach der überaus gelungenen und ausgedehnten Berichterstattung über die Polenreise des Papstes Johannes Pauls II. durch die ARD von „Blickfeld” nachträglich kleine Menschlichkeiten am Rande des großen Ereignisses herausgepickt ${ }^{21}$.

Warschaukorrespondent Peter Gatter zeigte Soutaneträger, Eis am Stiel lutschend, Nonnen, die Streuengelchen kontrollierten, Strohhütchen auf Priestertonsuren. Dann Jungen und Mädchen, die handgroße Kruzifixe über sich hielten, während der Papst predigte. Dazu indessen der fragwürdige Text: „Ein Kreuz, Symbol Christi, wird da zum bloßen Gegenstand, wo der Stellvertreter Christi auf Erden sichtbar wird.” Wer die Verhältnisse im Ostblock kennt, sollte doch wissen und textlich ausdrücken, daß es dieser Jugend um ein offenes Bekenntnis ging, das Kreuz durch die Kamera zum Fenster hinaus halten - nicht bloß als „Gegenstand”, sondern als Sinnbild des Glaubens im atheistisch geführten Staat.

\section{Programm ohne Kontrolle?}

Die Festlegung von „Kirche und Gesellschaft” auf die Magazinform läßt fraglich erscheinen, ob so die Gänze des kirchlichen Lebens erfaßt werden kann. Sicher nicht, wenn die Redaktion einseitige Ziele anpeilt, also, wie die Beispiele beweisen, Konflikt- 
stoffe bevorzugt, die keinesfalls repräsentativ für die Gesamtkirchen sind. In „Kirche und Gesellschaft" muß normale Kirche stattfinden können, und sie muß moderiert werden, ohne Partei zu nehmen.

Das verlangt der öffentlich-rechtliche Status der ARD. Was aber, wenn eine Sendereihe einseitig oder tendenziös geleitet wird? Hat etwa die Kirche eine Handhabe, dagegen vorzugehen? Hierzu äußerte sich Prälat Wilhelm Schätzler, Leiter des Referats „Hörfunk und Fernsehen” bei der Zentralstelle Medien der Deutschen Bischofskonferenz ${ }^{29}$ : Der Rundfunkrat habe die Programmkontrolle! „Von dieser gesetzlichen Gegebenheit gehen wir aus, da die Kirche als gesellschaftlich relevante Kraft dieses öffentlich-rechtliche Rundfunksystem mitträgt . . . Leider ziehen oft auch eklatante Verstöße gegen die Programmgrundsätze nahezu keine Folge nach sich. Die Verhaltensweisen der Verantwortlichen in den Rundfunkanstalten machen es einem daher schwer, auf dieses Funktionieren der Programmkontrolle zu vertrauen ${ }^{\prime \prime}$.

Auf die Angolasendung ${ }^{10}$ angesprochen bemängelte Schätzler, es sei nicht berücksichtigt worden, daß bei einem totalitären System verständlicherweise keine offenen Auskünfte bei den Betroffenen zu erhalten seien. „Daß auf der Basis dieser unzureichenden journalisischen Arbeit Wertungen vorgenommen wurden, ist ein zweiter Punkt, der Kritik erforderlich macht."

\section{Redakteure: Kritiker oder Verkünder?}

Schätzler wollte sich nicht auf diesen Einzelfall beschränken, sondern sprach von einer sich mehr und mehr ausbreitenden Mentalität in den Rundfunkanstalten: Werde Kritik geübt, rede man sofort von ideologischer Verfolgung, Zensur, Pression. „Daß schlechte journalistische Arbeit geleistet wurde, zu dieser Erkenntnis kommt keiner. Es wird daher notwendig sein, daß man über die Rolle des Journalisten im öffentlich-rechtlichen Rundfunk reflektiert und diese auch diskutiert."

Auf die Frage, wie er die Rolle der Kirchenfunkredakteure sehe (als Verkünder, als verlängerter Arm der Kirche, als ihr kritischer Begleiter), antwortete der Referatsleiter: „Die Kirchenfunkredakteure sind Mittler von religiös-kirchlichen Informationen und Mittler von in der Kirche vertretenen Meinungen. In dieser Position sind sie der Gesellschaft und damit auch der Kirche als relevanter Kraft dieser Gesellschaft verantwortlich." 23

\section{Das Recht der Kirchen}

Das „Blickfeld”-Magazin kann als Beispiel gelten für die unzureichende Präsenz der Kirche auch in manchen anderen Bereichen des Rundfunks. Es provoziert die Frage, wer schuld daran ist: Das System der Funkhäuser oder die damit befaßten Vertreter der Kirche?

Die bundesdeutschen Sender sind Anstalten des öffentlichen Rechts, das heißt, sie sind durch Gesetz gegründet und erfüllen bestimmte öffentliche Aufgaben unter staatlicher Aufsicht. Im „Fernsehurteil” ${ }^{4}$ bestimmt das Bundesverfassungsgericht, „daß dieses moderne Instrument der Meinungsbildung weder dem Staat noch einer gesellschaftlichen Gruppe ausgeliefert wird". In einem weiteren Urteil führt das Karlsruher Gericht aus, daß der Rundfunk nicht zuletzt infolge der Fernsehtechnik ,zu einem der mächtigsten Kommunikationsmittel und Massenmedien geworden ist, das wegen seiner weitreichenden Wirkungen und Möglichkeiten sowie der Gefahr des Mißbrauchs 
zum Zwecke einseitiger Einflußnahme auf die öffentliche Meinung nicht dem freien Spiel der Kräfte überlassen werden kann". Private Betreiber sind also in der gegenwärtigen Lage ausgeschlossen - ebenso wie die Errichtung eines eigenen katholischen Senders wie etwa in den Niederlanden.

Während das Zweite Deutsche Fernsehen den Kirchen ,angemessene Sendezeiten"26 für Gottesdienst und Weltdienst einräumt, enthält das WDR-Gesetz nur eine pauschale, kaum einklagbare Bestimmung ${ }^{27}$ : Der Sender hat die weltanschaulichen, wissenschaftlichen und künstlerischen Richtungen zu berücksichtigen. „Die sittlichen und religiösen Überzeugungen der Bevölkerung sind zu achten." Im Gegensatz zu den meisten anderen ARD-Sendern kennt das WDR-Gesetz offiziell keine kirchlichen Vertreter für den Rundfunkrat, das höchste Aufsichtsgremium. Nur für den Programmbeirat, der den Intendanten bloß beraten kann, darf die Kirche Mitglieder, „vorschlagen”. Allerdings sind im WDR-Rundfunkrat Geistliche über die CDU zu Sitz und Stimme gekommen, so der parteilose Prälat Prof. Dr. Johannes Overath und zwei evangelische Theologen. Diesen gesetzlichen Mangel zu beseitigen, müßte Aufgabe einer Novellierung sein. Die größte Anstalt mit den meisten ARD-Sendestunden muß den Kirchen als relevanten Gruppen eine Repräsentation im Rundfunkrat kraft Gesetzes zugestehen.

Katholische Medientheologen haben begründet, daß kirchliche Beiträge zum Fernsehprogramm besondere Ansprüche an die Verantwortungsaufteilung der Sender erfordern. „Die Kirche muß - wie jede andere gesellschaftliche Gruppe - zudem Gewicht darauf legen, daß sich Hörfunk und Fernsehen nicht als Instrumente der internen Opposition einer vielleicht verschwindenden Minderheit betätigen . . . Eine mit antiinstitutionellen Affekten besetzte Abteilung für kirchliche Sendungen und Themen ist sicher das Gegenteil des in diesem Bereich von der Sache her Geforderten." 28 Auch die „innere Rundfunkfreiheit” kann nicht dafür herhalten, daß Mitarbeiter ihre persönlichen Meinungen verbreiten und somit die gebotene Ausgewogenheit unterlaufen. Wer öffentlich-rechtlich kirchliche Sendungen veranstaltet, kann es nur in Zusammenarbeit mit kirchlichen Beauftragten.

\section{Die kirchlichen Beauftragten}

Wie weitreichend oder wie begrenzt sind die Befugnisse der kirchlichen Beauftragten? Wie groß ist ihr Einfluß gegenüber den Programmverantwortlichen? Haben die Kirchen überhaupt einen Rechtsanspruch auf öffentlich-rechtliche Kameras und Mikrophone?

Es ist zu unterscheiden zwischen Verkündigungssendungen und kirchenpolitischen, -informativen und -soziologischen Beiträgen, auf einen kurzen Nenner gebracht durch den Saarbrücker Intendanten Dr. Mai: zwischen „Heilsdienst” und „Weltdienst” der Kirchen. Die beiden christlichen Kirchen, die in jedem Funkhaus je einen theologischen Vertreter für Fernsehen und Hörfunk eingesetzt haben, sprechen entscheidend und verantwortlich beim Heilsdienst mit. „Die Verkündigungssendungen werden, unbeschadet der Programmverantwortung des Intendanten, von den kirchlichen Senderbeauftragten in Zusammenarbeit mit den Kirchenfunkredaktionen vorbereitet und von den Beauftragten theologisch verantwortet." ${ }^{29}$ Anders beim „Weltdienst”, den rein redaktionellen Beiträgen, vor allem im ARD-Gemeinschaftsprogramm. Hier können die kirchlichen Beauftragten nur beratend tätig werden, ohne theologisch verantwortlich zu sein. Ein Kontrollrecht steht den Beauftragten nicht zu, sie haben bloß eine „Initiativfunktion und einen redaktionellen Mitarbeiterstatus"29. Die Kontrollfunktion übt der Rundfunkrat aus. Mitglieder, die der Kirche gewogen sind und sie als relevante Kraft 
vertreten, können hier ihre Beanstandungen einbringen. Der bereits genannte Prälat Overath hat das verschiédene Male mit Erfolg tun können. Es ist auch möglich, daß eine alarmierte Öffentlichkeit kirchliche Benachteiligungen beseitigt. So gelang es einem Protest italienischer Pfarrer von Gastarbeitern, eine bereits abgesetzte Verkündigungssendung im 4. Kanal des WDR wieder aufleben zu lassen ${ }^{30}$.

Neue Fernsehtechniken geben den relevanten Kräften, die das Medium Rundfunk tragen, gewisse Chancen, das öffentlich-rechtliche Vorrecht oftmals „Monopol" genannt, zu ergänzen und Ungerechtigkeiten und Verstöße gegen den Pluralismus privat - zu eliminieren. Dabei kommt dem Kabelfernsehen größte Bedeutung zu.

\section{Anmerkungen}

' Indianersänger Floyd Westermann im ARD-„Blickfeld” vom 17.6.78.

2 Dietrich P. Winterberg, WDR.

${ }^{3}$ Thomas Schmitt, Uli Karp: Ein Priester geht stempeln, „Blickfeld” v. 21.1.78.

${ }^{4}$ Christian Modehn: Die Kirche im Dorf, „Blickfeld” v. 21.1.78.

${ }^{5}$ Christian Modehn: Katholische Kirche und Homosexualität, „Blickfeld” v. 18.3.78.

6 Winfried Schwamborn: Rassismus in Deutschland, „Blickfeld” v. 18.3.78.

${ }^{7}$ Winfried Schwamborn: Die EKD-Rassismus-Sonderrolle, „Blickfeld” v. 15.4.78.

${ }^{8}$ Peter Gatter: Billy Graham in Polen, u. Am Vorabend der Inthronisation des Papstes, „Blickfeld" v. 21.10.78.

9 Alberto Montani, Barbara Strauch, Jutta Uhl, Karl Unger: Der Konflikt der Katholischen Jungen Gemeinde mit der Deutschen Bischofskonferenz, „Blickfeld” v. 21.10.78.

10 Joachim Faulstich, Christiane Gerhards, Malte Rauch, Paul Staal: Katholische Kirche in Angola, „Blickfeld” v. 16.12.78.

11 Hermann A. Griesser: Wie kirchenkritisch darf 'Kirchenfernsehen' sein? in „Welt” vom 11.4.79.

12 Frank J. Heinemann: Blickfeld im Schußfeld in: „Frankfurter Rundschau” v. 19.4.79 sowie: Denen die Stimme leihen, die keine haben in: epd „Kirche und Rundfunk” v. 21.4.79.

13 Die Interessen unserer Partner sträflich verletzt: Missio-Erklärung in: epd „Kirche und Rundfunk" v. 13.6.79.

14 Hans-Jürgen Benedict: Stromzahlungsboykott, „Blickfeld” v. 18.2.78.

15 Winfried Schwamborn: Das Urteil des Bundesverfassungsgerichts, „Blickfeld” v. 20.5.78.

16 Ulrich Leinenweber: Der Verband der Christlichen Pfadfinder ..., „Blickfeld” v. 9.9.78.

17 Dietrich P. Winterberg: Nicaragua, „Blickfeld” v. 9.9.78.

18 N.N.: Kauft keine Früchte aus Südafrika, „Blickfeld” v. 17.3.79.

19 Lucas Maria Böhmer: Dieser Tod hat Gott nicht gefallen, „Blickfeld” v. 17.2.79.

20 Nina Gladitz: Ureinwohner in Australien, „Blickfeld” v. 21.4.79.

21 Peter Gatter: Polenbesuch des Papstes, „Blickfeld” v. 9.6.79. 
„Funk-Korrespondenz": Kann die katholische Kirche bei mißliebigen Sendungen überhaupt Stellung nehmen?, 23.5.79.

23 Wilhelm Schätzler: Aufgaben des Kirchenfunks, „Rheinischer Merkur” v. 11.5.79.

24 Urteil des Bundesverfassungsgerichts vom 28.2.1961.

25 Urteil des Bundesverfassungsgerichts vom 27.7.1971.

26 ZDF-Staatsvertrag $\$ 6$ Abs. 3.

27 WDR-Gesetz vom 25.5.1954, §4.

28 Prof. Karl Forster in: Essener Gespräche, S. 25, Aschendorff-Verlag, Münster.

29 P. Dr. Hermann-Josef Burbach msf, Katholischer Fernsehbeauftragter beim WDR, in: „Hinweise": Kirche und Rundfunk, Diözesanstelle Essen 4/1973.

30 Wieder geistliches Wort ... in: „Funk-Korrespondenz” Köln vom 11.7.1979.

\section{SUMMARY}

The organization of the German TV is neither governmental nor private. It has a religious department which produces a monthly magazine under the title "Vision, Church and Society". An analysis of the contributions to this magazine shows, that the editor of Westdeutscher Rundfunk (WDR) who is responsible for the transmission, is always trying to discover conflicts in Christian Churches and to evaluate them critically in view of the official Church. This has led to several protests particularly from the Catholic side. The public organization of the German radio stations prohibits tendencious and onesided Church criticism of that kind. Particularly because the Churches are part of public services carrying the broadcasting system, the editors cannot on their own authority transmit controversial topics only, but must give a balanced view of the whole Church, says Wilhelm Schätzler, in charge of radio and TV for the Bishops' Conference. He therefore requires that Church editors have to be mediators of religious news and of opinions as they are really represented in the Church. The two Christian Churches have a person responsible for radio and for TV at each station whose duty is to cooperate with the Church editors. In transmissions of a pastoral character they have a decisive and theologically responsible position. In contributions about the Church these Church responsibles should cooperate as consultants. The right to control a station is given to the supervising board, which for the WDR is the radio council, where Church representatives can voice their complaints.

\section{RÉSUMÉ}

La Télévision de la République Fédérale d'Allemagne, ni publique ni privée, mais une institution de droit public, dispose d'une rédaction de radio qui, sous le titre "tour d'horizon, Eglise et société" conteste un magazine, de façon mensuelle. Une analyse des articles montre que le rédacteur responsable des émissions de la WRD s'efforce presque sans exception de dépister des conflits dans les Eglises chrétiennes et de les exploiter selon la critique de l'Eglise officielle ce qui mène à des protestations surtout de Ia part des catholiques. L'état juridique et public interdit la partialité lorsque la critique devient visiblement tendentieuse à préjudice de l'Eglise dans la modération. Du fait que les Eglises aident à supporter les ondes en tant que forces importantes, les rédacteurs ne peuvent pas diffuser de leur propre chef des matières à conflit au lieu de l'ensemble ecclésiastique, postule Wilhelm Schätzler, responsable de la conférence des évêques allemands pour la radio et la télévision. Il exige que les rédacteurs d'émissions religieuses soient des émissaires des informations religieuses et des opinions représentées dans l'Eglise. 
Les deux Eglises chrétiennes ont attachés à chaque émetteur, un pour la radio et un pour la télévision, des responsables pour la collaboration avec les rédacteurs d'émissions religieuses. Ils ont une fonction décisive et théologiquement responsable pour les émissions d'apostolat. Dans les apports rédactionnels "sur" l'Eglise, ils conseillent les responsables ecclésiastiques. Le droit de contrôle revient aux conseils de surveillance, pour la WRD au conseil de radio, dans lequel des représentants de l'Eglise peuvent faire leurs plaintes.

\section{RESUMEN}

La Televisión en la República Federal de Alemania, que no es estatal ni privada, sino institución de derecho público, dispone de una redacción para temas eclesiales que emite cada mes un programa bajo el título "Punto de vista, Iglesia y sociedad". Un análisis de los programas muestra que los redactores responsables tratan de seguir la pista a conflictos latentes en las Iglesias y de juzgarlos críticamente, lo cual dió lugar a protestas, sobre todo de los católicos. El estatuto de la TV prohibe a los redactores unilateralidad tendenciosa o críticas negativas. Wilhelm Schätzler, representante de la Conferencia Episcopal Alemana ante la radio y la TV, opina que desde el momento que las Iglesias son elemento activo en el mantenimiento del sistema de radio y TV, los redactores no pueden emitir por su cuenta y riesgo temas conflictivos en lugar de ofrecer una panorámica eclesial. Los redactores de estos programas, añade, deben difundir informaciones religiosas y los criterios vigentes en las Iglesias.

Las dos grandes Iglesias del país tienen en las cadenas de radio y TV representantes que colaboran con los redactores en los programas religiosos. En los programas de carácter evangelizador tienen la última palabra sobre el contenido teológico. En los programas informativos sobre la Iglesia, los representantes de la jerarquía son simples consejeros. Controlan este trabajo los respectivos consejos de administración, ante los que pueden exponer sus quejas los representantes de la jerarquǐa. 